\title{
The Electric Field at the Chargeless Interface Between Two Regions of Space
}

\author{
Asim Gangopadhyaya \\ Loyola University Chicago, agangop@luc.edu \\ Robert McNees \\ Loyola University Chicago, rmcnees@luc.edu
}

Follow this and additional works at: https://ecommons.luc.edu/physics_facpubs

Part of the Physics Commons

\section{Recommended Citation \\ McNees, Robert A., and Asim Gangopadhyaya. "The Electric Field at the Chargeless Interface between Two Regions of Space." American Journal of Physics 82, no. 6 (June 1, 2014): 597-601. doi:10.1119/ 1.4867378 .}

This Article is brought to you for free and open access by the Faculty Publications and Other Works by Department at Loyola eCommons. It has been accepted for inclusion in Physics: Faculty Publications and Other Works by an authorized administrator of Loyola eCommons. For more information, please contact ecommons@luc.edu. Copyright @ 2014 American Institute of Physics. This article may be downloaded for personal use only. Any other use requires prior permission of the author and the American Institute of Physics. The following article appeared in McNees, Robert A., and Asim Gangopadhyaya. "The Electric Field at the Chargeless Interface between Two Regions of Space." American Journal of Physics 82, no. 6 (June 1, 2014): 597-601. doi:10.1119/1.4867378 and may be found at http://dx.doi.org/10.1119/1.4867378. 


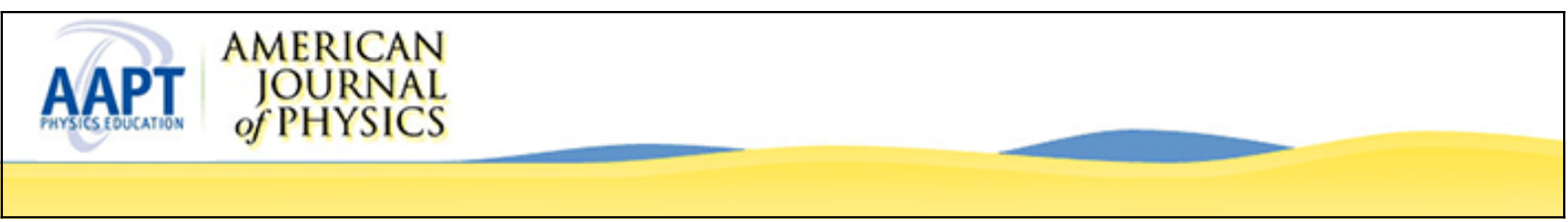

\section{The electric field at the chargeless interface between two regions of space}

Robert A. McNees and Asim Gangopadhyaya

Citation: American Journal of Physics 82, 597 (2014); doi: 10.1119/1.4867378

View online: http://dx.doi.org/10.1119/1.4867378

View Table of Contents: http://scitation.aip.org/content/aapt/journal/ajp/82/6?ver=pdfcov

Published by the American Association of Physics Teachers

\section{Articles you may be interested in}

Using Charge Distributions to "Immerse" Your Classroom in an Electric Field

Phys. Teach. 51, 234 (2013); 10.1119/1.4795370

Synchronous and Asynchronous Motors: Rotating Electric Fields

Phys. Teach. 47, 556 (2009); 10.1119/1.3246488

Causal equations for electric and magnetic fields and Maxwell's equations: Comment on a paper by Heras Am. J. Phys. 76, 101 (2008); 10.1119/1.2825390

Lab on Mapping Electric Fields

Phys. Teach. 44, L1 (2006); 10.1119/1.2221772

The Electric Fields Experiment: A New Way Using Conductive Tape

Phys. Teach. 44, 140 (2006); 10.1119/1.2173317

\section{WebAssign.}

Free Physics Videos

Add these videos and many more

resources - free with WebAssign.

bit.do/PhysicsResources

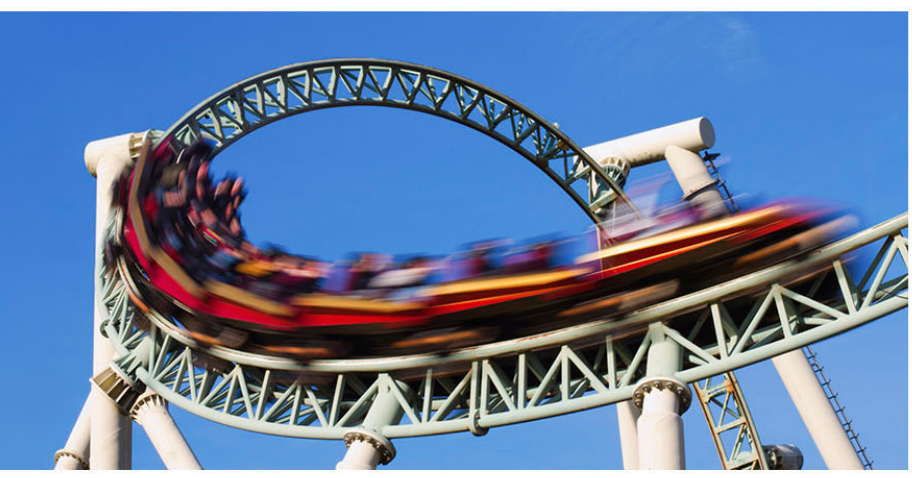




\title{
The electric field at the chargeless interface between two regions of space
}

\author{
Robert A. McNees ${ }^{\text {a) }}$ and Asim Gangopadhyaya ${ }^{\text {) }}$ \\ Department of Physics, Loyola University Chicago, Chicago, Illinois 60626
}

(Received 21 November 2012; accepted 20 February 2014)

\begin{abstract}
A common method for solving Poisson's equation in electrostatics is to patch together two or more solutions of Laplace's equation using boundary conditions on the potential and its gradient. Other methods may generate solutions without the need to check these conditions explicitly, and reconciling these solutions with the appropriate boundary conditions can be surprisingly subtle. As a result, a student may arrive at paradoxical conclusions-even in the case of elementary problems - that seem to be at odds with basic physical intuition. We illustrate this issue by showing how the potential of a uniformly charged ring appears to violate continuity of the normal component of the electric field at a chargeless surface. (C) 2014 American Association of Physics Teachers.

[http://dx.doi.org/10.1119/1.4867378]
\end{abstract}

\section{INTRODUCTION}

In electrostatics, the first step in solving Poisson's equation is often dividing space into multiple regions separated by two-dimensional interfaces. A few basic techniques are used to establish the solution (or its general form) in each region, and these solutions are then spliced together to give the full solution over all space. This last step is accomplished by imposing boundary conditions on the potential and its gradient at the interfaces between regions.

For example, suppose $\Phi_{1}(\vec{r})$ is the potential throughout a region $\mathcal{V}_{1}$ and $\Phi_{2}(\vec{r})$ is the potential in an adjacent region $\mathcal{V}_{2}$. If the two regions are separated by a surface $\mathcal{S}_{12}$ that carries a surface charge density $\sigma$, then the boundary conditions on the potentials are

$$
\begin{aligned}
& \left.\Phi_{1}(\vec{r})\right|_{\mathcal{S}_{12}}=\left.\Phi_{2}(\vec{r})\right|_{\mathcal{S}_{12}} \text { and } \\
& \left.\quad\left(\vec{\nabla} \Phi_{1}(\vec{r})-\vec{\nabla} \Phi_{2}(\vec{r})\right)\right|_{\mathcal{S}_{12}}=\frac{\sigma}{\epsilon_{0}} \hat{n}_{12}
\end{aligned}
$$

where $\hat{n}_{12}$ is the unit vector on $\mathcal{S}_{12}$ that points from $\mathcal{V}_{1}$ to $\mathcal{V}_{2}$ These conditions are usually summarized by stating that the potential is always continuous, but the normal component of the electric field will experience a "jump" discontinuity at a charged surface. This succinct explanation is quickly internalized by undergraduate students, and it forms an important part of their developing physical intuition for the behavior of the electric potential.

However, some techniques for solving Poisson's equation do not explicitly use these boundary conditions when determining the potential. For instance, given a charge distribution with azimuthal symmetry, it may be possible to obtain the solution on the symmetry axis directly from Coulomb's law. In regions where there is no charge this solution can be compared to the general solution of Laplace's equation to obtain a solution that is valid at points off the symmetry axis. $^{1,2}$ This solution might take different forms in different regions - inside and outside a charged sphere, for instanceand of course the appropriate boundary conditions must be satisfied at the interfaces between such regions. But in some cases, we find that verifying the boundary conditions and confirming the expected behavior of the potential can be surprisingly subtle. This is especially true in situations where there is no physical surface separating the regions. If there is no physical surface then there is no surface charge density, which leads one to expect that continuity of the electric field should be easy (or even trivial) to demonstrate. When this turns out to not be the case, students may be left wondering whether they have misunderstood the essential physical ideas expressed by the boundary conditions, or else made some sort of error in obtaining the potential. The purpose of this article is to show how the solutions of even very simple examples can be tricky to reconcile with the boundary conditions as they are expressed in Eq. (1).

In Sec. II, we establish notation and conventions by reviewing the boundary conditions on the potential for an azimuthally symmetric system that is most conveniently described in spherical coordinates. In subsequent sections we obtain the potential of a particular azimuthally symmetric system - a charged ring — using the method described above, and attempt to verify the boundary conditions as expressed in Eq. (1). As we will show, this requires both nontrivial effort and a careful re-examination of our assumptions.

\section{BOUNDARY CONDITIONS IN ELECTROSTATICS}

Let us determine the potential due to a charged spherical surface of radius $R$. The physical surface of the sphere naturally divides space into two distinct regions: the "inside" defined by $r<R$, and the "outside" defined by $r>R$. We will assume azimuthal symmetry, as is usually the case with problems in an undergraduate course, so the surface charge density $\sigma(\theta)$ depends only on the polar angle. Then the general solution for the potential $\Phi(r, \theta)$ in each region is given by

$$
\Phi(r, \theta)= \begin{cases}\Phi_{\text {in }}(r, \theta)=\sum_{\ell=0}^{\infty} a_{\ell} r^{\ell} P_{\ell}(\cos \theta) & \text { for } r<R \\ \Phi_{\text {out }}(r, \theta)=\sum_{\ell=0}^{\infty} \frac{b_{\ell}}{r^{\ell+1}} P_{\ell}(\cos \theta) & \text { for } r>R\end{cases}
$$

where $P_{\ell}(\cos \theta)$ are Legendre polynomials in the variable $\cos \theta$. The constants $a_{\ell}$ and $b_{\ell}$ are determined by imposing the following boundary conditions at the interface $r=R$ :

1. continuity of the potential,

$$
\Phi_{\text {in }}(R, \theta)=\Phi_{\text {out }}(R, \theta)
$$


2. a discontinuity in the radial derivative of the potential,

$$
\left.\frac{\partial \Phi_{\text {in }}}{\partial r}\right|_{R}-\left.\frac{\partial \Phi_{\text {out }}}{\partial r}\right|_{R}=\frac{\sigma(\theta)}{\epsilon_{0}} .
$$

The second boundary condition, which expresses the discontinuity in the radial component of the electric field due to a surface charge density on the physical surface $r=R$, is derived from the integral form of Gauss' law. It can also be obtained directly from the differential form of Maxwell's equations, as in Refs. 3-5.

The series solutions and boundary conditions for the potential, as written above, rely only on the symmetry of the charge distribution and the division of space into spherically symmetric inner and outer charge-free regions. Thus, they should be an appropriate starting point for other charge distributions with these properties, including situations where there is no physical surface separating the inner and outer regions. In Sec. III, we will consider the potential for just such an example and carefully analyze the boundary conditions at points where there is no surface charge.

\section{POTENTIAL DUE TO A UNIFORMLY CHARGED RING}

Let us consider a ring of radius $R$, oriented in the $x y$-plane and centered at the origin that carries a uniformly distributed total charge $Q$. This configuration is shown in Fig. 1. Working in spherical polar coordinates, space is once again naturally divided into the two domains $r<R$ and $r>R$. But unlike the case of the charged sphere, the interface $r=R$ between these regions is not a physical surface. The lefthand side of Eq. (4) is expected to vanish at most of the points on this interface, because there is no physical surface there to support a charge density $\sigma$.

The regions $r<R$ and $r>R$ contain no charge, and hence the potential in these domains is given by Eq. (2). For this type of azimuthally symmetric problem the coefficients $a_{\ell}$ and $b_{\ell}$ are determined by explicitly calculating the potential on the axis of symmetry (the $z$-axis with $\theta=0$ ) using Coulomb's law, expanding the result in powers of $r$ (for $r<R$ ) or $r^{-1}$ (for $r>R$ ), and then equating these expansions with the general solution, Eq. (2), evaluated at $\theta=0{ }^{6}$ The equality holds for all values of $r$ in each region, so the coefficients can be determined by a term-by-term matching of the expansions. Since this method gives the full solution in both regions without invoking the boundary conditions (3) and (4), one should check that the result exhibits the correct behavior at the interface $r=R$. Is the potential continuous, and is its radial derivative continuous at points where there is no charge?

The potential due to a uniformly charged ring, at a point $z=r$ on the axis of the ring, is given by

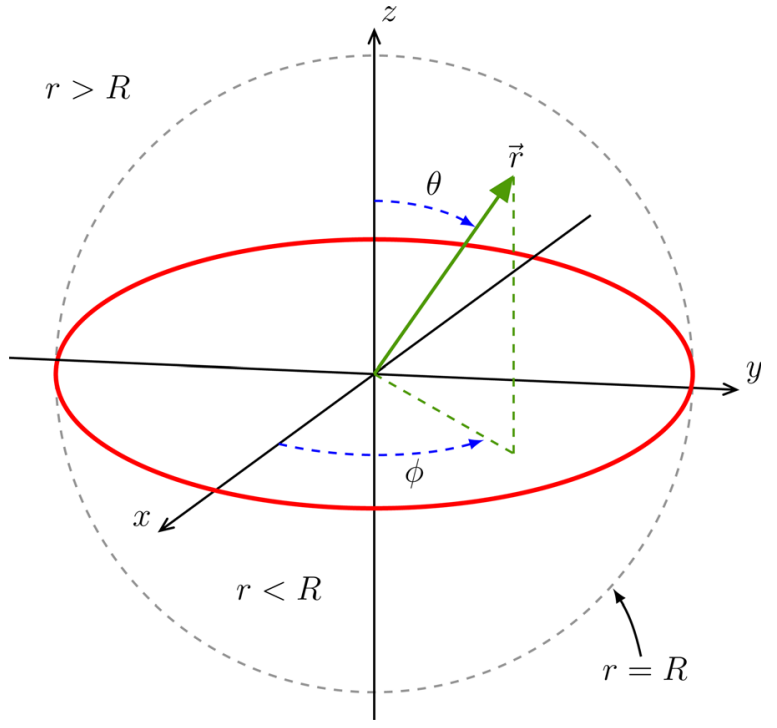

Fig. 1. A uniformly charged ring located at $r=R, \theta=\pi / 2,0 \leq \phi<2 \pi$. The dashed line represents the "surface" $r=R$ that separates the regions $r<R$ and $r>R$.

$$
\Phi(r, 0)=\frac{Q}{4 \pi \epsilon_{0} \sqrt{R^{2}+r^{2}}} .
$$

In the above two domains, this potential can be expanded as

$$
\Phi(r, 0)=\left\{\begin{array}{cc}
\frac{Q}{4 \pi \epsilon_{0}} \sum_{\ell=0}^{\infty}(-1)^{\ell}\left[\frac{(2 \ell) !}{2^{2 \ell}(\ell !)^{2}}\right] \frac{r^{2 \ell}}{R^{2 \ell+1}} \quad \text { for } r<R \\
\frac{Q}{4 \pi \epsilon_{0}} \sum_{\ell=0}^{\infty}(-1)^{\ell}\left[\frac{(2 \ell) !}{2^{2 \ell}(\ell !)^{2}}\right] \frac{R^{2 \ell}}{r^{2 \ell+1}} \quad \text { for } r>R
\end{array}\right.
$$

Comparing these expansions with the general solution in Eq. (2) shows that the odd coefficients $a_{2 \ell+1}$ and $b_{2 \ell+1}$ are all zero, while the even coefficients are

$$
\begin{aligned}
& a_{2 \ell}=\frac{(-1)^{\ell} Q}{4 \pi \epsilon_{0} R^{2 \ell+1}}\left[\frac{(2 \ell) !}{2^{2 \ell}(\ell !)^{2}}\right] \text { and } \\
& b_{2 \ell}=\frac{(-1)^{\ell} Q R^{2 \ell}}{4 \pi \epsilon_{0}}\left[\frac{(2 \ell) !}{2^{2 \ell}(\ell !)^{2}}\right] .
\end{aligned}
$$

Hence, the potential throughout the inner and outer regions can be written

$$
\Phi(r, \theta)= \begin{cases}\Phi_{\text {in }}(r, \theta)=\frac{Q}{4 \pi \epsilon_{0}} \sum_{\ell=0}^{\infty}(-1)^{\ell}\left[\frac{(2 \ell) !}{2^{2 \ell}(\ell !)^{2}}\right] \frac{r^{2 \ell}}{R^{2 \ell+1}} P_{2 \ell}(\cos \theta) & \text { for } r<R \\ \Phi_{\text {out }}(r, \theta)=\frac{Q}{4 \pi \epsilon_{0}} \sum_{\ell=0}^{\infty}(-1)^{\ell}\left[\frac{(2 \ell) !}{2^{2 \ell}(\ell !)^{2}}\right] \frac{R^{2 \ell}}{r^{2 \ell+1}} P_{2 \ell}(\cos \theta) & \text { for } r>R .\end{cases}
$$


A similar derivation of this potential can be found in Ref. 7, and an approximation motivated by numerical analysis is given in Ref. 8.

Now let us verify that the solutions $\Phi_{\text {in }}(r, \theta)$ and $\Phi_{\text {out }}(r, \theta)$ satisfy the boundary conditions at the interface $r=R$. Demonstrating continuity of the potential is straightforward: when evaluated at $r=R$ the two expressions in Eq. (8) are clearly equal to one another term-by-term. All that remains is to check whether the radial derivative of the potential is continuous on the parts of the interface $r=R$ where there is no charge. Computing the difference in the radial derivative across the interface, we find

$$
\begin{aligned}
& \left.\frac{\partial \Phi_{\text {in }}(r, \theta)}{\partial r}\right|_{R}-\left.\frac{\partial \Phi_{\text {out }}(r, \theta)}{\partial r}\right|_{R} \\
& =\frac{Q}{4 \pi \epsilon_{0} R^{2}} \sum_{\ell=0}^{\infty}(-1)^{\ell}\left[\frac{(2 \ell) !}{2^{2 \ell}(\ell !)^{2}}\right](4 \ell+1) P_{2 \ell}(\cos \theta) .
\end{aligned}
$$

According to Eq. (4), the left-hand side of this equation is expected to be zero at any point where there is no charge density (i.e., for $\theta \neq \pi / 2$ ). For instance, there is no charge along the $z$-axis, so the radial derivative of the potentials on the inner and outer regions should agree at $\theta=0$. But the difference in the radial derivatives at this point is

$$
\begin{gathered}
\left.\frac{\partial \Phi_{\text {in }}(r, 0)}{\partial r}\right|_{R}-\left.\frac{\partial \Phi_{\text {out }}(r, 0)}{\partial r}\right|_{R} \\
=\frac{Q}{4 \pi \epsilon_{0} R^{2}} \sum_{\ell=0}^{\infty}(-1)^{\ell}\left[\frac{(2 \ell) !}{2^{2 \ell}(\ell !)^{2}}\right](4 \ell+1) .
\end{gathered}
$$

Is the infinite sum on the right-hand side equal to zero? Applying one of the many convergence tests shows that the sum does not converge. ${ }^{9}$ Does this mean that the radial component of the electric field is somehow discontinuous at this point? Surely this cannot be the case-it is clear from Eq. (5) that the potential and its radial derivative are continuous everywhere on the $z$-axis.

To shed light on this confusing result, we return to the expression for the discontinuity in $\partial_{r} \Phi$ at a general point. Using $P_{2 \ell}(0)=(-1)^{\ell}(2 \ell) ! /\left[2^{2 \ell}(\ell !)^{2}\right]$, we write Eq. (9) as

$$
\begin{aligned}
& \left.\frac{\partial \Phi_{\text {in }}(r, \theta)}{\partial r}\right|_{R}-\left.\frac{\partial \Phi_{\text {out }}(r, \theta)}{\partial r}\right|_{R} \\
& =\frac{Q}{4 \pi \epsilon_{0} R^{2}} \sum_{\ell=0}^{\infty}(4 \ell+1) P_{2 \ell}(0) P_{2 \ell}(\cos \theta) .
\end{aligned}
$$

Since odd Legendre polynomials vanish when evaluated at 0 (i.e., $P_{2 \ell+1}(0)=0$ ) we can insert them in this expression to obtain a sum that includes both even and odd terms:

$$
\begin{aligned}
& \left.\frac{\partial \Phi_{\mathrm{in}}(r, \theta)}{\partial r}\right|_{R}-\left.\frac{\partial \Phi_{\mathrm{out}}(r, \theta)}{\partial r}\right|_{R} \\
& =\frac{Q}{2 \pi \epsilon_{0} R^{2}} \sum_{\ell=0}^{\infty}\left(\frac{2 \ell+1}{2}\right) P_{\ell}(0) P_{\ell}(\cos \theta) .
\end{aligned}
$$

This sum does not converge at $\theta=0$, as we saw above, or at any other value of $\theta .{ }^{10}$ However, using the completeness property of the Legendre polynomials, we obtain

$$
\begin{aligned}
\left.\frac{\partial \Phi_{\text {in }}(r, \theta)}{\partial r}\right|_{R}-\left.\frac{\partial \Phi_{\text {out }}(r, \theta)}{\partial r}\right|_{R} & =\frac{Q}{2 \pi \epsilon_{0} R^{2}} \delta(\cos \theta-0) \\
& =\frac{Q}{2 \pi \epsilon_{0} R^{2}} \frac{\delta(\theta-\pi / 2)}{|\sin (\pi / 2)|} \\
& =\frac{Q}{2 \pi \epsilon_{0} R^{2}} \delta\left(\theta-\frac{\pi}{2}\right) .
\end{aligned}
$$

The discontinuity in the radial derivative of the potential at $r=R$ is proportional to the Legendre series representation of the Dirac delta function. In fact, the right-hand side of this equation correctly expresses the uniform line charge on the ring, $\lambda=Q / 2 \pi R$, as a surface charge density. To the extent that we are willing to think of the delta function $\delta(\theta-\pi / 2)$ as being "zero" at $\theta \neq \pi / 2$, this confirms our expectation: the radial derivative of the potential is continuous at points where there is no surface charge.

On the other hand, a literal interpretation of Eq. (13) is problematic. A delta function is a distribution that makes sense only inside an integral, and its Legendre series representation, Eq. (12), does not converge. So how does one prove that the radial derivative of the potential is continuous at points on the surface $r=R$ where there is no charge, if expressions like Eqs. (12) and (13) cannot be evaluated at such points? The problem here is the condition given in Eq. (4), which assumes that the surface charge density is a wellbehaved function on the surface $r=R$. Since $\sigma$ is singular for the charged ring, we must take a step back and use the full integral form of Gauss' law to understand what happens.

Consider a Gaussian pillbox that straddles the surface $r=R$ and encloses a region $A$ on the surface. Then the integral form of Gauss' law gives

$$
\frac{1}{\epsilon_{0}} \int_{A} d a \sigma=\left.\int_{A} d a\left(\frac{\partial \Phi_{\text {in }}(r, \theta)}{\partial r}-\frac{\partial \Phi_{\text {out }}(r, \theta)}{\partial r}\right)\right|_{R},
$$

where $d a=R^{2} \sin \theta d \theta d \phi$ is the scalar area element on the sphere. The left-hand side is the charge $Q_{\text {enc }}$ enclosed by the Gaussian surface, which is just the surface charge on the part of the sphere $A$ inside the pillbox, and the right-hand side is the integral over $A$ of the divergent sum in Eq. (12). Let us explicitly evaluate this integral for arbitrary regions with $\theta_{1} \leq \theta \leq \theta_{2}$ and $\phi_{1} \leq \phi \leq \phi_{2}$, as shown in Fig. 2. Then Eq. (14) becomes

$$
Q_{\mathrm{enc}}=Q \frac{\Delta \phi}{4 \pi} \sum_{\ell=0}^{\infty}(2 \ell+1) P_{\ell}(0) \int_{\theta_{1}}^{\theta_{2}} d \theta \sin \theta P_{\ell}(\cos \theta),
$$

where $\Delta \phi=\phi_{2}-\phi_{1}$. The integral of $P_{\ell}$ can be expressed in terms of $P_{\ell+1}$ and $P_{\ell-1}$, and the resulting sum can be rearranged so that it takes the form

$$
Q_{\mathrm{enc}}=\left.Q \frac{\Delta \phi}{4 \pi}\left(\sum_{\ell=0}^{\infty}\left[P_{2 \ell+2}(0)-P_{2 \ell}(0)\right] P_{2 \ell+1}(\cos \theta)\right)\right|_{\theta_{1}} ^{\theta_{2}}
$$

Unlike the sum in Eq. (12), the sum in this expression is well-defined and converges for any value of $\theta$. It is just the Legendre series representation of a function $f(\theta)$ given by ${ }^{11}$ 


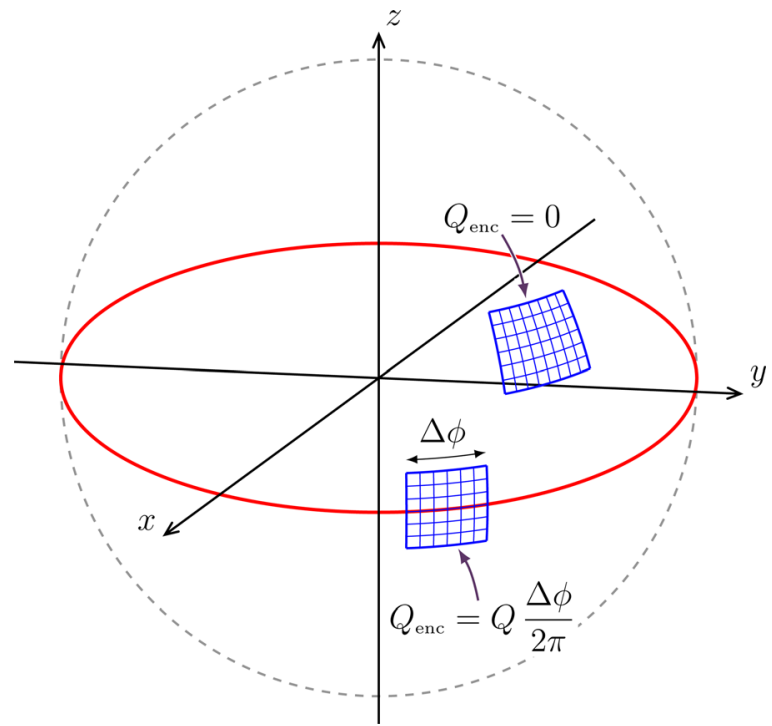

Fig. 2. Two patches $\theta_{1} \leq \theta \leq \theta_{2}, \phi_{1} \leq \phi \leq \phi_{2}$ on the surface $r=R$.

$$
f(\theta)=\left\{\begin{array}{cc}
-1 & \text { for } 0 \leq \theta<\pi / 2 \\
1 & \text { for } \pi / 2<\theta \leq \pi
\end{array}\right.
$$

As a result, the surface charge on the region $A$ is

$$
Q_{\mathrm{enc}}=\left\{\begin{array}{cc}
0 & \text { for } \theta_{1}<\theta_{2}<\pi / 2, \\
0 & \text { for } \pi / 2<\theta_{1}<\theta_{2}, \\
Q \frac{\Delta \phi}{2 \pi} & \text { for } \theta_{1}<\pi / 2<\theta_{2} .
\end{array}\right.
$$

Thus, the enclosed charge is always zero if $A$ does not overlap the ring at $\theta=\pi / 2$, and we can use this result to prove that the radial derivative of the potential is continuous at a point on $r=R$ with no surface charge. First, consider a small region $A$ that contains the point in question and does not overlap the ring. Then $Q_{\mathrm{enc}}=0$, and Eq. (14) becomes

$$
\left.\int_{A} d a\left(\frac{\partial \Phi_{\text {in }}(r, \theta)}{\partial r}-\frac{\partial \Phi_{\text {out }}(r, \theta)}{\partial r}\right)\right|_{R}=0 .
$$

This equality does not depend on the details of $A$-it is true for all values $\theta_{1}, \theta_{2}, \phi_{1}$, and $\phi_{2}$ such that $A$ does not overlap the ring-so we can conclude that the integrand is equal to zero. Therefore, the radial derivative of the potential is continuous at points on the surface $r=R$ where there is no charge.

\section{CONCLUSION}

Using the example of a uniformly charged ring, we showed that verifying the expected behavior of the potential at the interface between two charge-free regions can be nontrivial. In particular, significant analysis and careful consideration of Gauss' law is needed to confirm that the derivative of the potential is continuous at points on the interface where there is no charge. Similar issues arise in many other situations involving singular or localized charge distributions, such as a collection of point charges, multiple charged rings, or a band or annulus of charge. It is important to be aware of instances where these elementary examples appear to disagree with a student's physically motivated expectations.

The potential for the uniformly charged ring was obtained without appealing to the boundary conditions at $r=R$, so the process of checking these conditions might be regarded as an intellectual exercise rather than a necessary step in solving the problem. But it is easy to see how the example we have described might cause confusion for students in their first electromagnetism course. Methods for obtaining solutions like Eq. (8) are usually taught not long after the boundary conditions are first expressed in the form given in Eq. (1), and it is natural for a student to appeal to these simple boundary conditions as a tool for understanding new results. Examples like the one described here both reinforce the physical meaning of the boundary conditions - the electric field is continuous at points where there is no charge-and illustrate how careful one must be in jumping from a generally applicable result like Gauss' Law to an expression like Eq. (1).

One might question the relevance of this example, since a perfectly thin ring of charge is unrealistic. Shouldn't the issues we describe be resolved by more physically realistic charge distributions like the ones shown in Fig. 3? After all, the sum in Eq. (10) fails to converge because the charge distribution of the ring is singular, with the surface charge density given by a delta function:
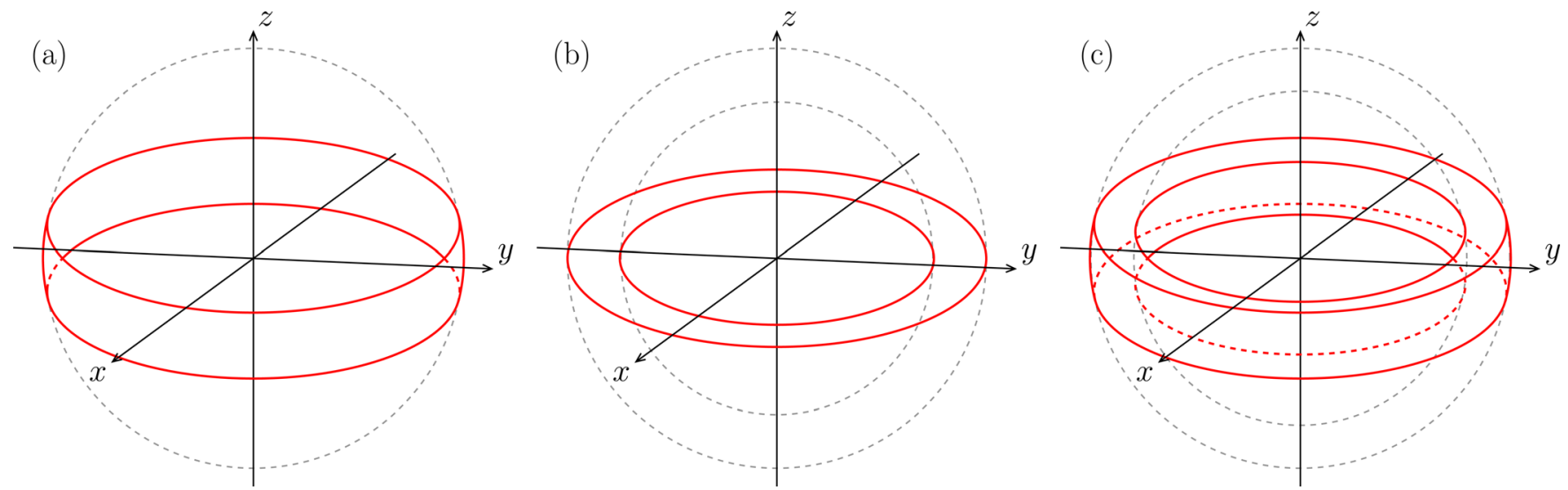

Fig. 3. Three ways of smoothing out the singular charge distribution of the ring: (a) a charged band $(\pi / 2-\delta<\theta<\pi / 2+\delta, r=R$ ); (b) a charged annulus $\left(\theta=\pi / 2, R_{\text {inner }}<r<R_{\text {outer }}\right)$; and (c) a solid of revolution formed by rotating an annular sector about the $z$-axis $\left(\pi / 2-\delta<\theta<\pi / 2+\delta, R_{\text {inner }}<r<R_{\text {outer }}\right)$. In each case, the analog of Eq. (12) converges but demonstrating continuity of the radial component of the electric field at points with $\sigma=0$ requires nontrivial identities for Legendre polynomials. 


$$
\sigma(\theta)=\frac{\lambda}{R} \delta\left(\theta-\frac{\pi}{2}\right) .
$$

While it is true that spreading the charge over an extended region addresses the issue of convergence, there are at least two reasons why our example should not be discounted. The first reason is pedagogical. Singular charge distributions are a common tool in electromagnetism courses because they tend to be conceptually simple and mathematically tractable, and this helps a student focus on essential physics. When one of these examples leads to a confusing result, the student is forced to analyze basic statements like Eq (1) and think deeply about the physics involved in order to determine the source of the problem. The second reason is technical, but no less important. Replacing the singular charged ring with one of the distributions shown in Fig. 3 makes it more difficult to demonstrate continuity of the gradient of the potential. In those cases, verifying the boundary conditions requires Legendre polynomial identities that are outside the scope of most undergraduate courses. $^{12}$ Such examples, which are more difficult to work out in detail, would be just as likely to leave students wondering why their expression for the potential does not seem to satisfy the expected boundary conditions at points where $\sigma=0$.

\section{ACKNOWLEDGMENTS}

The authors wish to thank the referees of an early version of this manuscript for several helpful suggestions that improved the quality of the paper. A.G. would like to thank the Center for Experiential Learning at Loyola University Chicago for an Engaged Learning Faculty Fellowship that provided partial support for this research. R.M. is supported by a Faculty Research Stipend from Loyola University Chicago.

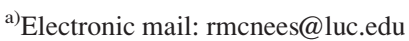

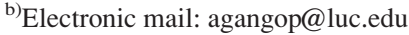

${ }^{1}$ J. D. Jackson, Classical Electrodynamics, 3rd ed. (John Wiley \& Sons, New York, 1999).

${ }^{2}$ D. J. Griffiths, Introduction to Electrodynamics, 3rd ed. (Prentice-Hall, Englewood Cliffs, NJ, 1991).

${ }^{3}$ S. T. Epstein, "Alternative discussion of discontinuities at an interface," Am. J. Phys. 53, 583-584 (1985).

${ }^{4}$ D. G. Hall, "A few remarks on the matching conditions at interfaces in electromagnetic theory," Am. J. Phys. 63, 508-512 (1995).

${ }^{5}$ These references assume that the surface charge density $\sigma$ is a wellbehaved function on the surface.

${ }^{6}$ Note that $P_{\ell}(\cos 0)=P_{\ell}(1)=1$ for all $\ell$.

${ }^{7}$ E. A. Matute, "On the vector solutions of Maxwell equations in spherical coordinate systems," Rev. Mex. Fis. 51, 31-36 (2005); available at: http:// arxiv.org/abs/physics/0512261.

${ }^{8}$ F. Zypman, "Off-axis electric field of a ring of charge," Am. J. Phys. 74, 295-300 (2006).

${ }^{9}$ For instance, Stirling's approximation $\ell ! \sim \ell^{\ell} e^{-\ell} \sqrt{2 \pi \ell}$ can be used to verify that the summand grows as $\sqrt{\ell}$ for large $\ell$, and therefore does not vanish in the $\ell \rightarrow \infty$ limit. Combining subsequent even $(\ell=2 k)$ and odd $(\ell=2 k+1)$ terms in the sum gives a strictly negative summand proportional to $1 / \sqrt{k}$ at large $k$. This form of the sum can be shown to diverge by the integral test or by comparison with the harmonic series.

${ }^{10} \mathrm{~A}$ thorough discussion of the convergence properties of the Legendre series solution for the potential of a uniform charged ring can be found in F. Glück, "Axisymmetric electric field calculation with zonal harmonic expansion," Prog. Electromagn. Res. 32, 319-350 (2011).

${ }^{11}$ Students will have encountered a similar Legendre series in problem 3.22 of Ref. 2.

${ }^{12}$ One must use identities for Legendre polynomials on the half-intervals $0<\theta<\pi / 2$ and $\pi / 2<\theta<\pi$, where the familiar orthogonality conditions do not apply.

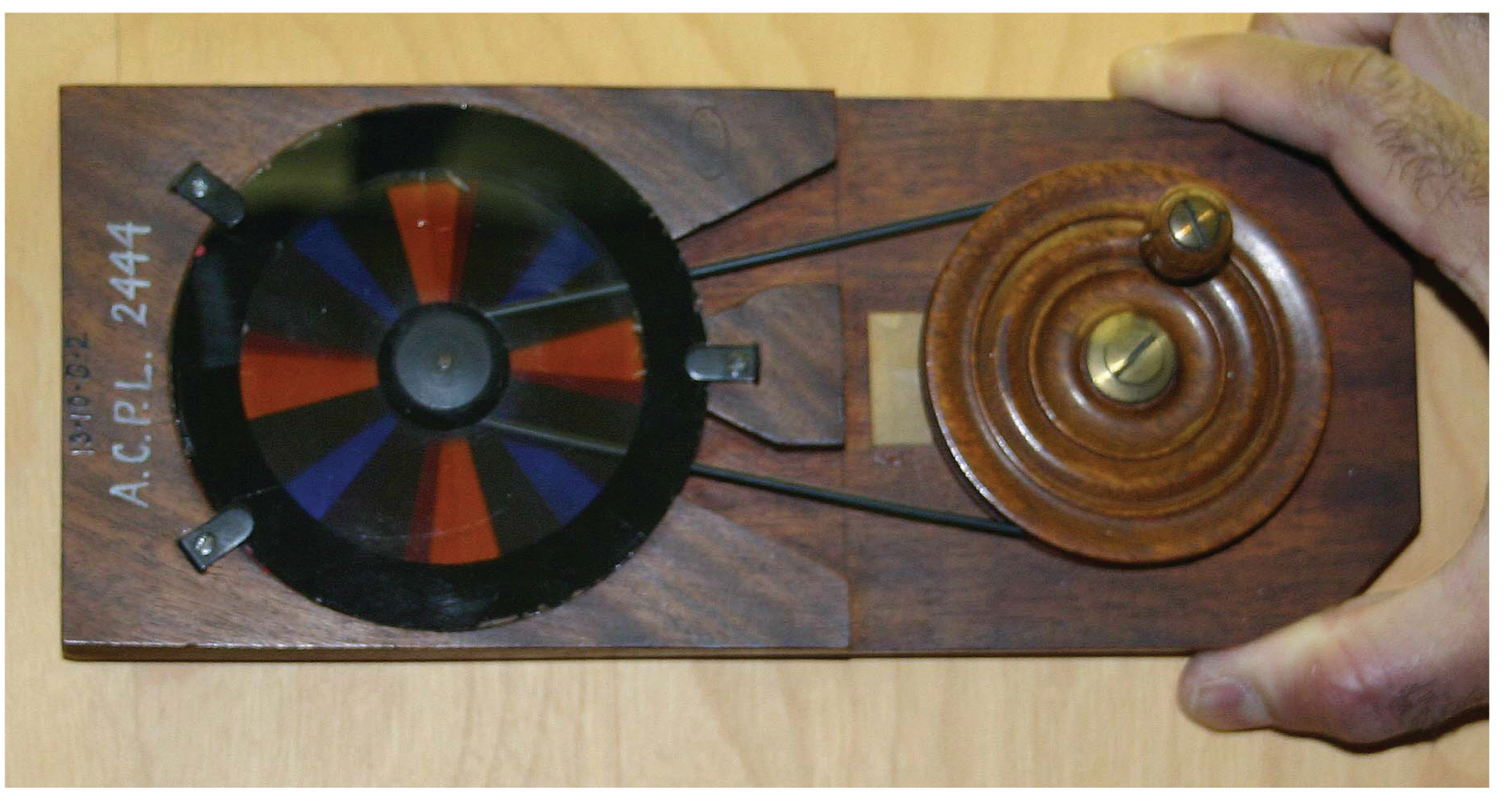

Newton's Color Wheel

In 1882, Walker Hall on the Amherst College campus burned, and with it all of the Philosophical Cabinet. This Color Wheel, designed for use in a projector, was one of many pieces purchased from the Paris firm of Ducretet to replace the lost apparatus. It is listed at 30 francs (about \$5.50) in the contemporaneous Ducretet catalogue. (Notes and photograph by Thomas B. Greenslade, Jr., Kenyon College) 